\title{
A family with Von Hippel-Lindau disease with different presentations
}

\author{
Ching-lin Wong, ${ }^{1,2}$ Ming-Yueh Lee, ${ }^{1}$ Sunder R., ${ }^{1}$ Jamalia R., ${ }^{1}$ \\ Karunakar T.V.N., ${ }^{1}$ Joseph A. ${ }^{1}$ \\ 'Department of Ophthalmology, Kuala Lumpur Hospital, Malaysia; ${ }^{2}$ Department of \\ Ophthalmology, Penang Hospital, Malaysia
}

\begin{abstract}
Objective: To report three cases of Von Hippel-Lindau (VHL) disease from a family with different presentations.

Method: Case series.

Results: Case 1, a 14-year-old Malay boy with a history of gradual blurring of vision in both eyes presented with sudden deterioration of right vision. Fundus examination revealed bilateral retinal capillary hemangioma with exudative retinal detachment. His right eye underwent combination therapy of laser photocoagulation, cryotherapy, intravitreal anti-VEGF and photodynamic therapy, but failed to improve vision. His left vision deteriorated and eventually required surgical intervention. Case $\mathbf{2}$ was the sister of case 1. She was a 17-year-old Malay girl who presented with sudden onset of left eye pain and redness. Diagnosis of left eye rubeotic glaucoma with closed funnel retinal detachment secondary to a huge retinal hemangioma was made. She underwent left eye external drainage of subretinal fluid plus anterior retinal cryopexy. The rubeotic vessel regressed postoperatively and IOP reduced. Case 3 was the eldest sister of case 1; a 19-year-old Malay girl who came for eye screening after two of her siblings were diagnosed with VHL. She was, however, asymptomatic. Fundus examination revealed right retinal capillary hemangioma. She was treated with laser photocoagulation and her condition remained stable. Systemic investigations identified midline cystic masses in the brain, spine and pancreas. Conclusions: This case series highlight different clinical pictures of Von Hippel-Lindau disease. As marked visual loss is a dreadful sequela of VHL, it is important to screen the family members as early detection and management of ocular and systemic lesions save sight and life.
\end{abstract}

Key words: Von Hippel-Lindau, retina capillary hemangioma

\section{Introduction}

Von-Hippel Lindau (VHL) disease is a rare hereditary disorder that results from an inherited mutation in the VHL tumor suppressor gene and is characterized by retinal and central nervous system hemangioblastoma. It is panethnic, and inherited in an autosomal dominant manner and occurs in one in 36,000 births per year. It is also associated with renal cell carcinoma, phaeochromocytoma, renal, pancreatic and epididymal cyst. ${ }^{1}$ Retinal capillary hemangioma $(\mathrm{RCH})$ is the most frequent

Correspondence: Dr. Ming-Yueh Lee, Consultant Ophthalmologist \& Glaucoma Specialist, Department of Ophthalmology, Kuala Lumpur Hospital, 50586 Jalan Pahang, Kuala Lumpur, Malaysia. E- mail: leemingyueh@yahoo.com 
and often the earliest manifestation of VHL disease. It occurs in about $37 \%$ of patients with clinically definite and genetically confirmed VHL. ${ }^{2}$ Forty-two percent of patients have unilateral $\mathrm{RCH}$ and $58 \%$ of have bilateral $\mathrm{RCH} .{ }^{3}$ Most $\mathrm{RCH}$ enlarge progressively; and the retinal changes can be various.

We report three cases of $\mathrm{VHL}$ from a family with different presentations.

\section{Case 1}

A 14-year-old Malay boy presented with gradual reduction of vision in both eyes over two years prior to presentation. On examination, his best-corrected visual acuity (BCVA) was $6 / 60$ in the right eye and $6 / 15$ in the left eye. Both eyes had normal anterior segments. Fundus examination of the right eye revealed a solitary $\mathrm{RCH}$ at the temporal region. Dilated and tortuous retinal vessels present were associated with subretinal lipid deposition and exudative retinal detachment involving the macular (Figs. 1a \&1b). A similar fundus finding was noted in the left eye with the solitary RCH in the infero-temporal peripheral retina (Figs. 2a \& 2b). Systemic examination was unremarkable. Screening tests for other systemic associations were negative.
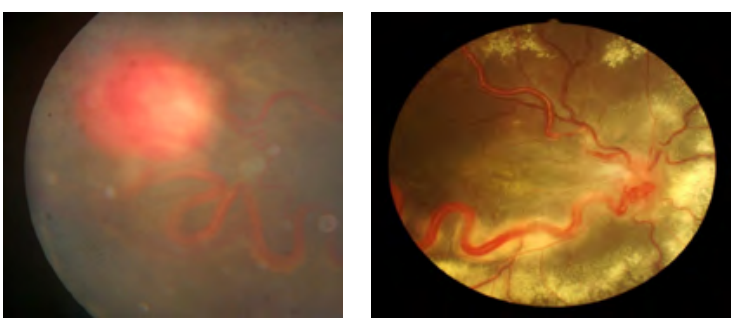

Figs. 1a \& 1b. Fundus photos of the right eye show dilated and tortous feeder vessel with marked retinal edema and lipid exudates. An orange red solitary $\mathrm{RCH}$ is located at the temporal periphery retina.
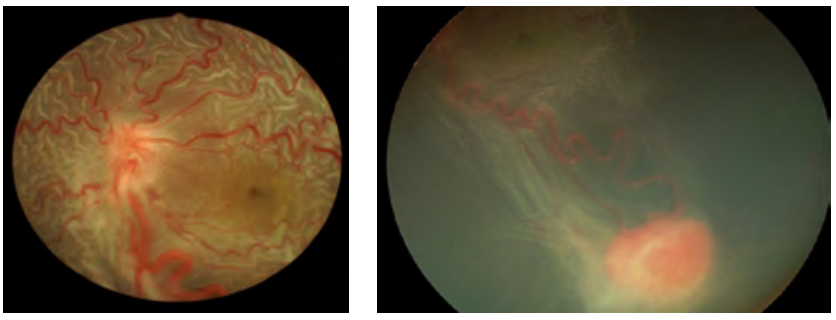

Figs. 2a \& 2b. Fundus photos of the left eye show extensive exudative retinal detachment. $\mathrm{RCH}$ at the inferotemporal peripheral retina associated with exudative and tractional retinal detachment.

Both eyes were treated with a combination of laser indirect ophthalmoscopy, peripheral retinal cryopexy, intravitreal Ranibizumab $0.5 \mathrm{mg}$ (Lucentis, Genetech) and subtenon injection of triamcinolone $(40 \mathrm{mg})$. There was an initial responce to treatment in the right eye with partial resolution of the exudative detachment and BCVA improved to $6 / 18$ (Fig. 3). However, the exudative detachment then recurred (Figs. $4 a \& 4 b$ ). The left eye did not show any response with progressive exudative retinal detachment. A repeat of the treatment regime in both eyes was unsuccessful. He was then given photodynamic therapy to the $\mathrm{RCH}$ in both eyes, which unfortunately was accompanied by extensive exudative retinal detachment and choroidal effusion. 

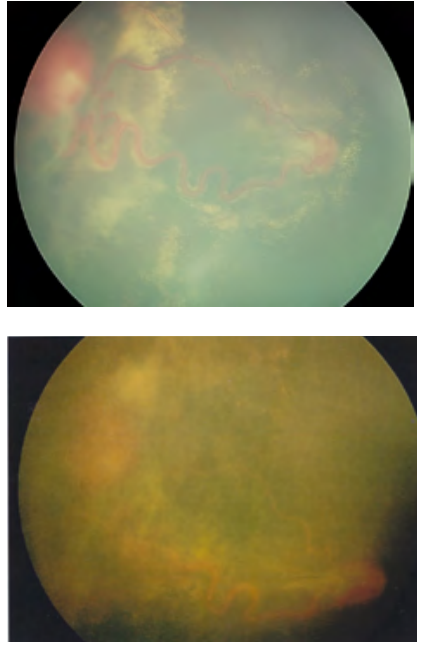

Fig. 3. Fundus photo of the right eye shows reduction of subretinal lipid exudation over the posterior pole.

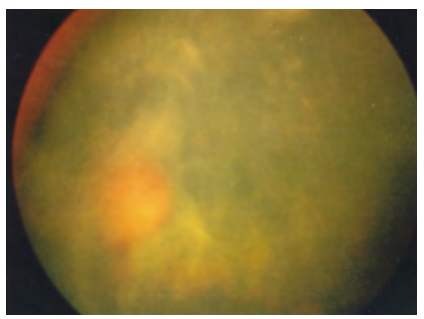

Figs. 4a \& 4b. Fundus photos of the right eye show increased gliosis and posterior pole exudation.

Oral Prednisolone $15 \mathrm{mg}$ OD $(0.5 \mathrm{mg} / \mathrm{kg})$ was started in addition to another intravitreal injection of Ranibizumab $0.5 \mathrm{mg}$ and subtenon triamcinolone ( $40 \mathrm{mg}$ ). The exudation became less in the right eye and the retina was flat six weeks later. However, his right eye vision remained poor. His left eye developed cataract with total retinal detachment (Fig. 5). He underwent left eye lens aspiration with scleral buckling, pars plana vitrectomy, subretinal fluid drainage, C3F8 gas tamponade combined with cryotherapy and endophotocoagulation. Unfortunately, his vision deteriorated to no perception of light due to extensive fibrosis and tractional retinal detachment (Figs. 6a \& 6b).

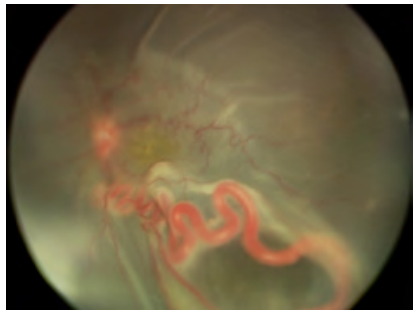

Fig. 5. Fundus photo of the left eye shows total funnel retinal detachment.
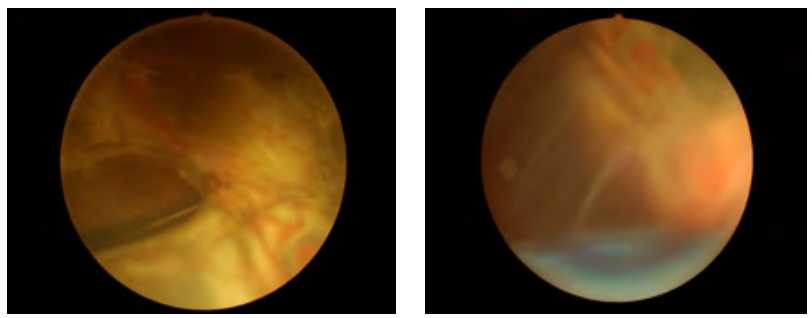

Figs. 6a \& 6b. Fundus photos of the left eye show extensive fibrosis and tractional retinal detachment. 


\section{Case 2}

A 17-year-old Malay girl, a sister of case 1, presented with sudden onset of left eye pain and redness for two days. She complained of left eye blurring of vision for many years. BCVA of the right eye was $6 / 9$ and perception of light in the left eye. Ocular examination showed left circumciliary injection with edematous cornea. There was 360-degree of rubeosis iridis and ectropion uveal with intraocular pressure (IOP) of $58 \mathrm{mmHg}$. Fundal examination of the left eye revealed closed funnel retinal detachment. Examination of the right eye was normal. Her IOP was controlled with systemic and topical antiglaucoma medication preoperatively. She underwent left eye transcleral subretinal fluid drainage with anterior retinal cryopexy. A huge retinal hemangioma was discovered (Fig. 7) after the retina was flattened intraoperatively (Fig. 8). She was diagnosed to have left rubeotic glaucoma with retinal detachment secondary to VHL. Post operatively, IOP was reduced and the rubeosis regressed (Fig. 9). Systemic examination and investigations were unremarkable.
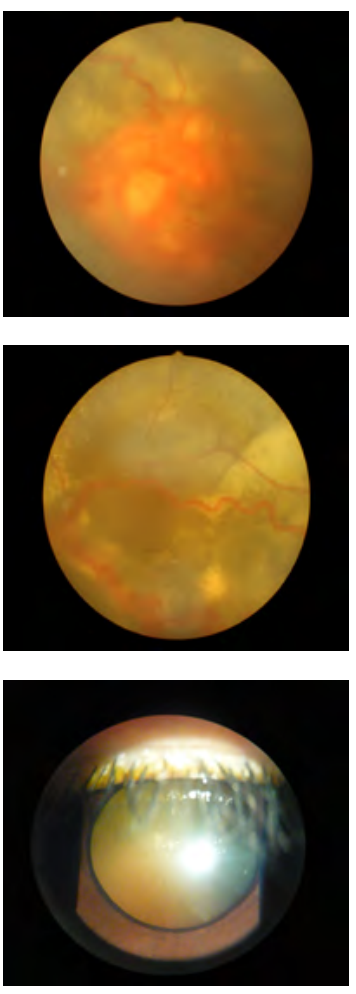

Fig. 7. Huge RCH post scleral drainage.

Fig. 8. Flattening of funnel retinal detachment.

Fig. 9. Regression of rubeosis iridis.

\section{Case 3}

A 19- year-old Malay girl, eldest sister of case 1, came for eye screening after two of her siblings were diagnosed to have $\mathrm{RCH}$. She was asymptomatic. Her BCVA was $6 / 6$ both eyes. Anterior segments for both eyes were normal. Fundus examination 
showed a right solitary retinal capillary hemangioma about two-disc diameter at nasal peripheral retina (Fig. 10). She was treated with argon laser photocoagulation. After two sessions of laser photocoagulations, the lesion shrunk and became whitish in color (Fig. 11). MRI brain, spine and abdomen identified midline cystic masses in the brain, lesion at spine and pancreas. She was then referred to neurosurgery and a medical team for further management.
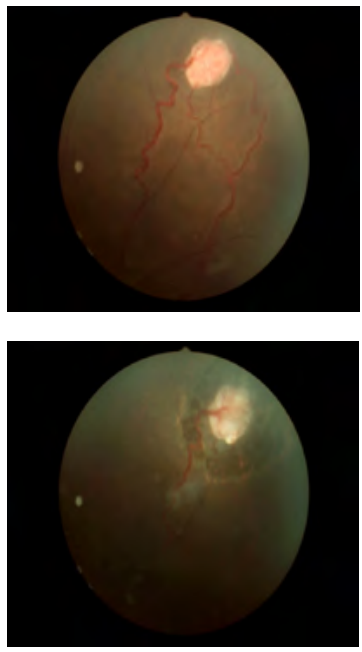

Fig. 10. $\mathrm{RCH}$ located at the nasal peripheral retina.

Fig. 11. $\mathrm{RCH}$ appeared whitish in colour post laser treatment.

\section{Discussion}

Von-Hippel Lindau disease is a blinding disease and $\mathrm{RCH}$ is the most frequent and the earliest manifestation. Von-Hippel Lindau gene mutation carriers have a 35\% probability of visual loss by age 50 .

The natural course of untreated $\mathrm{RCH}$ is uncertain. Most eyes with $\mathrm{RCH}$ show progression over time, although some may remain stable or regress spontaneously. The exudate tends to accumulate in the macular region when the tumor increases in size. The initial cystoid macular edema often progresses to a distinct star-shaped exudative maculopathy. It may further complicate with macular gliosis; from which the angioma could extend through a break in the internal limiting membrane into the vitreous. If left untreated; the serous and lipid exudation from the hemangioma can lead to total retinal detachment. Although rare, retinal and iris neovascularisation can follow, and potentially lead to secondary glaucoma and phthisis bulbi. ${ }^{45}$

Retinal changes due to $\mathrm{RCH}$ can be divided into various stages by Vail's classification: ${ }^{6}$

Stage I: Early stage with dilation of feeding artery and draining vein and angioma formation;

Stage II: Development of hemorrhages and exudation;

Stage III: Massive exudation and retinal detachment;

Stage IV: Uveitis, absolute glaucoma, and loss of the eye. 
There are two possible mechanisms contributing to visual loss: Subretinal exudation due to leakage from the hemangioblastoma and traction retinal detachment due to fibrovascular proliferation at the vitreoretinal interface. ${ }^{7}$ Causes of visual loss in VHL are cystoid macular edema, subretinal exudates, exudative or tractional retinal detachment, vitreous hemorrhage or neovascular glaucoma ${ }^{4}$ as presented in this series. In view of the progressive nature of subretinal exudation in VHL, most authorities agree that treatment should be initiated as soon as a $\mathrm{RCH}$ is detected.

The choice of treatment method is dictated by media clarity, lesion size, location and the retina changes. Various treatment modalities including observation, laser photocoagulation, cryotherapy and vitreoretinal surgery, have been described. ${ }^{8}$ The possibility that the upregulation of downstream genes, such as VEGF leading to the formation of vascular tumors has prompted the exploration of anti-angiogenic therapies as a possible treatment modality for ocular VHL. ${ }^{9}$ Recent case reports have also suggested that anti-VEGF agent, delivered either systemically ${ }^{10}$ or via intravitreal injection may be useful. ${ }^{11,12}$

Management of $\mathrm{RCH}$ is mainly orientated toward reducing the destructive exudation associated with these lesions. For early-detected cases like our Case 3 direct Argon laser photocoagulation showed a response rate of $91-100 \%$. Laser photocoagulation is most effective in tumors that are $1.5 \mathrm{~mm}$ or smaller and is effective in $\mathrm{RCH}$ up to $4.5 \mathrm{~mm}$ in size. It can be applied directly to the tumor, to the feeder artery or to both. Change of color of the tumor from bright red to pale pink, and narrowing of vessels are indicative of adequate response to treatment; which normally show in four to six weeks. Usually more than one treatment session is required to be effective. ${ }^{13}$

Visual prognosis is often poor and management is difficult when patients come in advanced stages like the case 1 in these case series. We used combined laser photocoagulation and cryotherapy in view of the huge size and the anterior locality of the $\mathrm{RCH}$ with a significant amount of subretinal fluid. Cryotherapy was applied until the ice ball completely enclosed the $\mathrm{RCH}$ as described by Welch. ${ }^{14}$ Cryotherapy can be repeated two months after the previous application. ${ }^{15}$ Complex vitreoretinal surgery including vitrectomy, endolaser and combined scleral buckling is usually required for a larger $\mathrm{RCH}$ complicated with retinal detachment. However treatment of huge tumor could be hazardous and the visual outcome is often disappointing. Marked increase in subretinal exudation and a total retinal detachment has resulted in this patient's left eye after treatment.

Clinical studies also reported elevated intraocular levels of VEGF in patients with VHL disease leading to the formation of vascular tumors. ${ }^{7,9}$ This has substantiated the use of anti-VEGF to achieve regression of the RCH and subretinal exudation. ${ }^{11}$ However it did not work so well in our patient although there was some evidence of resorption of subretinal exudation. We suggest more prospective clinical trials to evaluate the efficacy of this treatment modality.

Photodynamic therapy (PDT) with verteporfin has been reported as a treatment for RCH in VHL. It has been hypothesized that as verteporfin targets vascular endothelial cells, there may be selective affinity for verteporfin in the leading 
pathological capillaries of hemangioma. There are several cases of retinal angiomas having encouraging results after being treated with PDT. ${ }^{16,17}$ Bakri et al. reported one case where retinal hemangioma was successfully closed after verteporfin PDT along with argon laser and TTT. However, the patient developed a combined rhegmatogenous and exudative retinal detachment with proliferative vitreoretinopathy that needed subsequent vitrectomy, scleral buckling and silicone oil tamponade. ${ }^{18}$ Our case 1 patient had extensive exudative retinal detachment with choroidal effusion post PDT treatment could possibly explained by upregulation of VEGF caused by PDT. PDT may not be an effective treatment and further investigations are required to determine the indications and parameter for effective treatment of RCH with PDT.

Rubeosis iridis and neovascular glaucoma are uncommon anterior segment complications of VHL; only $2 \%$ cases are reported. ${ }^{1}$ It usually leads to painful and phthisical eyes that require enucleation. The aim of the treatment was to save the eye and provide some symptomatic relieve. In our case 2 , the rubeosis did regress and IOP reduced following treatment.

$\mathrm{RCH}$ could be the only manifestation of VHL disease. Therefore, patients with an isolated hemangioma should be screened for VHL disease. A systemic workup should include MRI or CT of the head, upper cervical spinal cord, and abdomen, as well as a urinalysis to investigate for the presence of catecholamines in the urine. Genetic testing was not done. Ideally it should have been done to detect the genetic mutation in this family with subsequent genetic counselling.

\section{Conclusion}

This case series highlight the different presentations and different stages of VHL and the complexity in clinical management. Treatment of $\mathrm{RCH}$ is difficult when the patient presents late and the visual prognosis is usually poor. We also highlight the importance of screening the family members for early treatment in order to save sight and save life.

\section{References}

1. Webster ARF, Maher ERMDF, Moore ATF. Clinical Characteristics of Ocular Angiomatosis in von Hippel-Lindau Disease and Correlation With Germline Mutation. Arch Ophthalmol 1999;117(3):371-378.

2. Wong WTMDP, et al. Genotype-Phenotype Correlation in von Hippel-Lindau Disease With Retinal Angiomatosis. Arch Ophthalmol 2007;125(2):239-245.

3. Wong T, et al. Clinical Characterization of Retinal Capillary Hemangioblastomas in a Large Population of Patients with von Hippel-Lindau Disease. Ophthalmology 2008;115(1):181-188.

4. Aumiller MS. Juxtapapillary hemangioma: a case report and review of clinical features and management of von Hippel-Lindau disease. Optometry - Journal of the American Optometric Association 2005;76(8):442-449.

5. EB A. Von Hippel-Lindau disease. In:Thomann KH, Marks ES, Adamczyk DT (Eds.), Primary eyecare in systemic disease. $2^{\text {nd }}$ ed. New York:McGraw-Hill 2001.

6. Vail D. Angiomatosis retinae, eleven years after diathermy coagulation. Amer J Opthalmol 1958;46: 525-534. 
7. McDonald H. Diagnostic and Therapeutic Challenges in Von Hippel Lindau disease. The Journal of Retinal and Vitreous Diseases 2007;27(8):1142-1147.

8. Singh $A D$, et al. Treatment of retinal capillary hemangioma. Ophthalmology 2002;109(10):1799-1806.

9. Rosenblatt Ml, Azar DT. Anti-angiogenic therapy: prospects for treatment of ocular tumors. Seminars in Ophthalmology 2006;21(3):151-160.

10. Girmens J-F, et al. Treatment of von Hippel-Lindau retinal hemangioblastoma by the vascular endothelial growth factor receptor inhibitor SU5416 is more effective for associated macular edema than for hemangioblastomas. Amer J Ophthalmol 2003;136(1):194-196.

11. Wong WT, et al. Intravitreal Ranibizumab Therapy for Retinal Capillary Hemangioblastoma Related to von Hippel-Lindau Disease. Ophthalmology 2008;115(11):1957-1964.e3.

12. Michels S, et al. [Intravitreal anti-VEGF therapy for capillary hemangioblastomas in von HippelLindau disease]. Klin Monatsbl Augenheilkd 2008;225(4):292-294.

13. Schmidt $D$, Natt E, Neumann HPH. Long-term results of laser treatment for retinal angiomatosis in von Hippel-Lindau disease. Eur J Med Res 2000;5:47-58.

14. Welch RB. Von Hippel-Lindau disease: the recognition and treatment of early angiomatosis retinae and the use of cryosurgery as an adjunct to treatment. Trans Am Ophthalmolo Soc 1970;68: 367-424.

15. Shields J. Response of retinal capillary hemangioma to cryotherapy. Arch Ophthalmol 1993;111:551.

16. Bhattacharjee H, Deka H, Deka S et al. Verteporfin Photodynamic Therapy of Retinal Capillary Hemangioblastoma in von Hippel-Lindau disease. Indian Journal of Ophthalmology, 2010. Occurs in one of 58(1):73-75

17. Neal HA. Retinal C apillary Hemangioma Treated with Verteporfin Photodynamic Therapy. American Journal Of Ophthalmology, 2002;134:788-790.

18. Bakri SJ, Sears J, Singh A. Transient Closure of a Retinal Capillary Hemangioma with Verteporfin Photodynamic Therapy. Retina, 2005. 25(8):1103-1104. 